\title{
Gitelman's Syndrome: An unusual cause of Recurrent Attack of Quadriparesis
}

\author{
SABRINA SHAHRIN,${ }^{1}$ RAKESH PANDAY,${ }^{2}$ KAMAL UDDIN SOHEL, ${ }^{3}$ NAZMUL ISLAM,${ }^{2}$ MOTLABUR RAHMAN, ${ }^{4}$ AKM \\ AMINUL HOQUE ${ }^{5}$
}

\begin{abstract}
:
Gitelman's syndrome, discovered in 1966 by Gittleman,Graham and Welt, is an autosomal recessive renal tubular disorder and characterized by hypokalemic metabolic alkalosis, hypomagnesaemia, hypocalciuria.'This is a rare cause of hypokalemia which has an autosomal recessive inheritance. Here we are reporting a case of a 35 years old lady presented with recurrent attacks of quadriparesis due to hypokalemia and pain in multiple joints. Later we diagnosed the case as Gitelman's syndrome.
\end{abstract}

Key words: Gitelman's syndrome, quadriparesis

\section{Introduction:}

Gitelman's syndrome is primarily a renal tubular disorder of thiazide sensitive $\mathrm{Na}-\mathrm{Cl}$ co-transporter. The clinical feature of this syndrome are muscle cramp, fatigue, muscle weakness, carpopedal spasm, paralysis and very rarely may cause respiratory failure and cardiac complication. ${ }^{2}$ Another outcome seen is chondrocalcinosis in multiple joints secondary to hypomagnesia. ${ }^{3}$ This rare disorder affects both male \& female. ${ }^{1}$ Often there will be ahistory of patients' sibling with Gitelman syndrome and parents have no disease. The main differential diagnosis is to consider is Bartter syndrome. ${ }^{4}$ The biochemical \&clinical features are almost similar. Gitelman syndrome is usually diagnosed during adolescent and adulthood \& clinical symptoms are rarely apparently before the age of 5 . On the other hand Bartter syndrome presents early childhood with severe failure to thrive and growth retardation. ${ }^{5}$ We can differentiate these two conditions by doing urinary calcium level. In Giteleman syndrome there will be hypocalciuria and in Bartter syndromethere will be normocalciuria.

\section{Case report:}

A 33 year-old-female was admitted in the dept. of Medicine with the complaints of recurrent attacks of severe generalized weakness for 1 year, difficulty in sitting down \& stand up spontaneously for 1 year, low back pain \& pain in both knee $\&$ thigh for 1 month. 1 year back she developed severe

1. IMO, Dept. of Medicine, Dhaka Medical College \& Hospital.

2. HMO, Dept. of Medicine, Dhaka Medical College \& Hospital.

3. Assistant Registrar, Dept. of Medicine, Dhaka Medical College \& Hospital.

4. Assistant Professor,Dept. of Medicine, Dhaka Medical College \& Hospital.

5. Associate Professor, Dept. of Medicine, Dhaka Medical College \& Hospital.

Correspondence : Dr. Sabrina Shahrin. Indoor Medical Officer (Medicine Unit-VII), Dhaka Medical College \& Hospital. Mob: 01711890998. E-mail: sharna188@hotmail.com. generalized weakness for which she was unable to do her daily work. She also complained of difficulty in sitting down $\&$ standing up from sitting position occasionally. She also complained of low back pain\& pain in both knees \& ankles for a month. She didn't give any history of vomiting or passage of loose stool; rather she gave history of constipation for which she used laxatives. There was no history of heat intolerance, weight loss, palpitation, polyuria, polydypsia \& also no significant family history. On examination, she was ill looking, pulse- 100/m, BP- $80 / 60 \mathrm{~mm}$ $\mathrm{Hg}$. All other general \& systemic examination was normal.

CBC revealed $\mathrm{Hb}-12 \mathrm{~g} / \mathrm{dl}, \mathrm{ESR}-50 \mathrm{~mm}$ in $1 \mathrm{st} \mathrm{hr}$, TC- 10000/ cmm, N- 67\%, L-28\%, E- 04\%, Platelet- 400000/cmm. RBS$5.4 \mathrm{mmol} / \mathrm{L}, \mathrm{S}$. Creatinine - $1.3 \mathrm{mg} / \mathrm{dl}$. She had persistent hypokalemia on different occasions varying from 1.8 to 2.9 $\mathrm{mmol} / \mathrm{L}$. MRI of Brain showed prominent cerebral cortical sulci \& MRI of Cervical spine showed early degenerative disc disease.

S. Magnesium was $1.2 \mathrm{mg} / \mathrm{dl}$, S. Osmolality $-278 \mathrm{mosmol} / \mathrm{kg}$, S. Calcium - 9.9 mg/dl, S. Albumin - $42 \mathrm{~g} / \mathrm{dl}$, urine R/E normal. 24 hours urinary electrolytes showed $\mathrm{Na}-260 \mathrm{mmol} /$ $\mathrm{d}(\mathrm{N}=40-220), \mathrm{K}-74.7 \mathrm{mmol} / \mathrm{d}(\mathrm{N}=25-125), \mathrm{Cl}-288 \mathrm{mmol} / \mathrm{d}$ $(\mathrm{N}=110-250)$, Calcium $8 \mathrm{mg} / \mathrm{d}(\mathrm{N}=100-300)$.

\section{Discussion:}

Causes of hypokalemia can be divided into four main categories; inadequate intake, GIT loss, renal loss, and excess potassium shift from extracellular to intracellular fluid. ${ }^{2}$ As our patient was complaining neck pain, at first we excluded neck abnormalities by doing an X-ray and MRI of the neck. Both the investigations showed degenerative disc disease. But her real problem, sudden attack of weakness of four limbs remained unsolved. She was persistently hypokalemic. There was no history of vomiting, diarrhea, anorexia nervosa or any significant drug intake and pseudohypokalemia was excluded by $\mathrm{CBC}$ report which showed no extreme rise of leucocytes. She had no clinical signs or symptoms of thyroid 
abnormality. We could not evaluate her thyroid status due to her financial constrain. At this point we discharged the patient and further investigations were done on OPD basis. Her serum calcium level was normal but her serum magnesium level was low. We did the urinary electrolytes level, both spot and 24 hours. Both the reports showed normal potassium excretion but slightly raised sodium excretion. At last we did urinary calcium excretion rate which was low. For later age of presentation along with other clinical and biochemical features we labeled the patient as a case of Gitelman's Syndrome. Unfortunately we could not do the gene testing due to lack of facilities.

Antenatal Bartter syndrome, classical Bartter syndrome and Gitelman's syndrome are the three phenotypes of Bartter syndrome that has now been recognized. ${ }^{4}$ Gitelman's syndrome is an autosomal recessive renal tubular disorder resulting from loss of function mutation in the SLC12A3 gene encoding the thiazide sensitive $\mathrm{NaCl}$ co-transporter located on chromosome $16 \mathrm{q} 3$. $^{3}$ It is common in adolescent age group with an incidence of 1-2 per million. ${ }^{3}$ It is not as infrequent as reflected in the literature because in the past it has been confusedwith Bartter syndrome from which it is distinguished by a milder clinical picture, absence of polyuria and biochemical evidence of hypomagnesaemia, hypocalciuria and later age of presentation. But now it can be easily diagnosed by molecular DNA diagnostic studies which shows mutations in the NCCT gene. ${ }^{3}$

Rodriguez-Soranio were the first to suggest that hypocalciuria may be used to differentiate between Gitelman's syndrome and classic Bartter syndrome. But our current understanding of tubular function does not easily explain the dissociation of calcium and magnesium excretion in these disorders. ${ }^{4}$ The thick ascending limb of loop of Henley is the major site for magnesium reabsorption, wherethereabsorption is thought to be parallel to the calcium reabsorption. Consequently involvement of thick ascending loop would be expected to promote severe magnesium wasting which is not usually present in classic Bartter syndrome. Paradoxically in Gitelman's syndrome there is more consistent and severe magnesium wasting which would not be expected from a tubular defect limited to the distal convoluted tubule. ${ }^{4}$

Therapeutic options in Gitelman's syndrome are limited. Supplementation of potassium and magnesium is the cornerstone of treatment. Thus asymptomatic stable hypokalemia and borderline hypomagnesemia is the therapeutic goal. ${ }^{6}$ We treated our patient with potassium sparing diuretics spironolactone. ${ }^{3}$ We advised her to take sufficient salt (as low $\mathrm{NaCl}$ supply exaggerates volume depletion and increases the secondary hyperaldosteronism resulting in intense hypokalemia), restricted the intake of licorice, lemon juice, iced tea etc. Our patient is doing well now. High dose of indomethacin may be indicated where there is growth retardation. ${ }^{3}$ Rofecoxib has been used successfully by Marvan et al.

Overall the prognosis of GS is good in terms of renal function and maintaining growth. But there are some case reports renal dysfunction like proliferative nephritis, diffuse messangial hypercellularity and focal segmental glomerulosclerosis. ${ }^{1}$ The cause of renal damage is thought to be due to prolonged RAAS stimulation and release of TGB-B, TNF-a and other cytokines. ${ }^{1}$ Our patient also has degenerative disc disease but no features of chondrocalcinosis were found. There are case reports that hypomagnesemia may cause chondrocalcinosis. ${ }^{7}$

The diagnosis of Gitelman's syndrome is important as profound hypokalemic paralysis patient having GS reported increasingly in Asia.

\section{Conflict of interest: None.}

\section{References:}

1. Pandey BD, Pedneker JS, Chavan AS, Korivi D, Shah KA, Kulkarni PU.A case of Nephrotic syndrome with Gitelman's syndrome. JAPI2010; 58: 10-12.

2. Eren AM, Tabur S, Sczgin B, Scbuncu T.A rare case of hypokalemia: Gitelman's syndrome. European Journal of General Medicine 20011; 8(2): 154-6.

3. Akhtar N, Hafeez F. A rare case of Gittelman's Syndrome with hypophosphatemia. Journal of college of physicians and surgeons, Pakistan 2004; 19(4): 257-259.

5. Chowta KN, Chowta NM. Gitelman's syndrome. Indian Journal of Human Genetics 2006; 12(1): 17-19.

4. Sylva S, Nevman D, Lnenicka P, Stekrova J.Gitelman's syndrome as a cause of psychomotor retardation in a toddler. Arab journal of Nephrology and Transplantation. 2013; 6(1): 37-9.

6. Knobel U, Modarrus G, Schneerran M, Schmid C. Gitelman's syndrome with persistent hypokalemia. Journal of Medical Case Reports 2011; 5(312): 9.

7. Tomas MA, Cid RA, Fenandaz CC, Catala CJ.Carpal arthritis as the initial manifestation of Gitelman's syndrome Rheumatologoa Clinica. 2012; 159(1): 8. 